\title{
DYNAMIC VISION
}

From Images to Face Recognition 
This page is intentionally left blank 


\title{
DYNAMIC VISION From Images to Face Recognition
}

\author{
Shaogang Gong \\ Queen Mary and Westfield College \\ Stephen J McKenna \\ University of Dundee \\ Alexandra Psarrou \\ University of Westminster
}




\author{
Published by \\ Imperial College Press \\ 57 Shelton Street \\ Covent Garden \\ London WC2H 9HE
}

\title{
Distributed by
}

World Scientific Publishing Co. Pte. Ltd.

5 Toh Tuck Link, Singapore 596224

USA office: 27 Warren Street, Suite 401-402, Hackensack, NJ 07601

UK office: 57 Shelton Street, Covent Garden, London WC2H 9HE

\section{British Library Cataloguing-in-Publication Data}

A catalogue record for this book is available from the British Library.

First published 2000

Reprinted 2001, 2005

\section{DYNAMIC VISION \\ From Images to Face Recognition \\ Copyright $\odot 2005$ by Imperial College Press}

All rights reserved. This book, or parts thereof, may not be reproduced in any form or by any means, electronic or mechanical, including photocopying, recording or any information storage and retrieval system now known or to be invented, without written permission from the Publisher.

For photocopying of material in this volume, please pay a copying fee through the Copyright Clearance Center, Inc., 222 Rosewood Drive, Danvers, MA 01923, USA. In this case permission to photocopy is not required from the publisher.

ISBN $\quad 1-86094-181-8$

Printed in Singapore by Mainland Press 
To my Parents and Aunt Mae

Shaogang Gong

To Collette

Stephen McKenna

'To my Parents

Alexandra Psarrou 
This page is intentionally left blank 


\section{Contents}

Preface $\quad x v$

PART I BACKGROUND 1

1 About Face 3

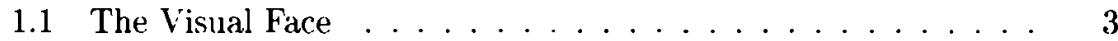

1.2 The Changing Face . . . . . . . . . . . 4

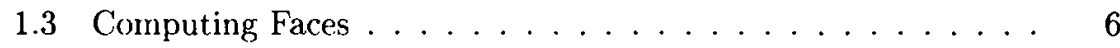

1.4 Biological Perspectives . . . . . . . . . . . . . . 11

1.5 The Approach . . . . . . . . . . . . . 12

2 Perception and Representation 15

2.1 A Distal Object. . . . . . . . . . . . . . . . 16

2.2 Representation by 3D Reconstruction . . . . . . . . 18

2.3 Two-dimensional View-based Representation . . . . . . . . 19

2.4 Image Template-based Representation . . . . . . . . . . . . 21

2.5 The Correspondence Problem and Alignment . . . . . . . . 22

2.6 Biological Perspectives . . . . . . . . . . . . . . . 26

2.7 Discussion . . . . . . . . . . . . . . . . . . . 29

3 Learning under Uncertainty 31

3.1 Statistical Learning . . . . . . . . . . . . . . . . 32

3.2 Learning as Function Approximation . . . . . . . . . 33

3.3 Bayesian Inference and MAP Classification . . . . . . . . 36

3.4 Learning as Density Estimation . . . . . . . . . . . . . 37

3.4.1 Parametric Models . . . . . . . . . . . . . . . 37

3.4 .2 Non-parametric Models . . . . . . . . . . . . . . 39

3.4 .3 Semi-parametric Models . . . . . . . . . . . . . . 40

3.5 Unsupervised Learning without Density Estimation . . . . . . 41 
3.5.1 Dimensionality Reduction . . . . . . . . . . . . 43

3.5 .2 Clustering . . . . . . . . . . . . . . . 43

3.6 Linear Classification and Regression . . . . . . . . . 44

3.6.1 Least-squares . . . . . . . . . . . . . . . 45

3.6.2 Linear Support Vector Machines . . . . . . . . . . . 45

3.7 Non-linear Classification and Regression . . . . . . . . . 48

3.7.1 Multi-layer Networks . . . . . . . . . . . . . . 48

3.7 .2 Support Vector Machines . . . . . . . . . . . 50

3.8 Adaptation . . . . . . . . . . . . . 51

3.9 Biological Perspectives . . . . . . . . . . . . . . . 53

3.10 Discussion . . . . . . . . . . . . . . 54

Part il From Sensory to Meaningful Perception 57

4 Selective Attention: Where to Look 59

4.1 Pre-attentive Visual Cues from Motion . . . . . . . . . . . 60

4.1.1 Measuring Temporal Change . . . . . . . . . . 61

4.1 .2 Motion Estimation . . . . . . . . . . . . . . . 62

4.2 Learning Object-based Colour Cues . . . . . . . . . . . 65

4.2 .1 Colour Spaces . . . . . . . . . . . . . . . 66

4.2 .2 Colour Density Models . . . . . . . . . . . . 68

4.3 Perceptual Grouping for Selective Attention . . . . . . . . 71

4.4 Data Fusion for Perceptual Grouping . . . . . . . . . . . 73

4.5 Temporal Matching and Tracking . . . . . . . . . . 76

4.6 Biological Perspectives . . . . . . . . . . . . . 77

4.7 Discussion . . . . . . . . . . . . . . . 78

5 A Face Model: What to Look For 81

5.1 Person-independent Face Models for Detection . . . . . . . 82

5.1.1 Feature-based Models . . . . . . . . . . . . . 82

5.1 .2 Holistic Models . . . . . . . . . . . . . . 83

5.1 .3 The Face Class . . . . . . . . . . . . . . . . . 85

5.2 Modelling the Face Class . . . . . . . . . . . . . 86

5.2.1 Principal Components Analysis for a Face Model . . . . 87

5.2.2 Density Estimation in Local PCA Spaces . . . . . . . . 88

5.3 Modelling a Near-face Class . . . . . . . . . . . . . . 89

5.4 Learning a Decision Boundary . . . . . . . . . . . . . 90 
5.4.1 Face Detection in Dynamic Scenes . . . . . . . . . 91

5.4 .2 Normalisation. . . . . . . . . . . . . . . . . . . . . 93

5.4.3 Face Detection using Multi-layer Perceptrons . . . . . . 95

5.4.4 Face Detection using Support Vector Machines . . . . . 97

5.5 Perceptual Search _ . . . . . . . . . . . . . . . . 99

5.6 Biological Perspectives . . . . . . . . . . . . . . . . . 101

5.7 Discussion . . . . . . . . . . . . . . . . . 102

6 Understanding Pose 103

6.1 Feature and Template-based Correspondence . . . . . . . . 105

6.2 The Face Space across Views: Pose Manifolds . . . . . . . . . . 105

6.3 The Effect of Gabor Wavelet Filters on Pose Manifolds . . . . . 111

6.4 Template Matching as Affine Transformation . . . . . . . 113

6.5 Similarities to Prototypes across Vicws . . . . . . . . . . . 118

6.6 Learning View-based Support Vector Machines . . . . . . . . 121

6.7 Biological Perspectives . . . . . . . . . . . . . . . . . . 123

6.8 Discussion . . . . . . . . . . . . . . . . 123

7 Prediction and Adaptation $\quad 125$

7.1 Temporal Observations . . . . . . . . . . . . . 128

7.2 Propagating First-order Markov Processes . . . . . . . . . . . 129

7.3 Kalman Filters . . . . . . . . . . . . . . . . 131

7.4 Propagating Non-Gaussian Conditional Densities . . . . . . 132

7.4.1 Learning Priors using HMMs and EM . . . . . . . . 132

7.4.2 Observation Augmented Density Propagation . . . . . . 133

7.5 Tracking Attended Regions . . . . . . . . . . . . 134

7.6 Adaptive Colour Models . . . . . . . . . . . . . . . 136

7.7 Selective Adaptation . . . . . . . . . . . . . . . 140

7.8 Tracking Faces $\ldots \ldots \ldots \ldots \ldots \ldots \ldots$

7.9 Pose Tracking . . . . . . . . . . . . . . . . . 147

7.9.1 Person-specific Pose Tracking . . . . . . . . . . . . . 149

7.9 .2 Person-independent Pose Tracking . . . . . . . . 150

7.10 Biological Perspectives . . . . . . . . . . . . . . . 156

7.11 Discussion . . . . . . . . . . . . . . . 157 
PART III Models of Identity 161

8 Single-View Identification $\quad 163$

8.1 Identification Tasks . . . . . . . . . . . . . . . . . 163

8.2 Nearest-neighbour Template Matching . . . . . . . . . . . . 165

8.3 Representing Knowledge of Facial Appearance . . . . . . . 166

8.4 Statistical Knowledge of Facial Appearance . . . . . . . . 168

8.4.1 Low Dimensionality: Principal Components Analysis . . 168

8.4.2 Separability: Linear Discriminant Analysis . . . . . . 173

8.4.3 Sparseness and Topography: Local Feature Analysis . . 176

8.5 Statistical Knowledge of Identity . . . . . . . . . . . . 176

8.5.1 Identification Tasks Revisited . . . . . . . . . . . 177

8.5.2 Class-conditional Densities for Modelling Identity . . . 179

8.6 Structural Knowledge: The Role of Correspondence . . . . . 180

8.6.1 Beyond Alignment: Correspondence at a Single View . 181

8.6.2 Combining Statistical and Structural Models . . . . . 182

8.7 Biological Perspectives . . . . . . . . . . . . . 183

8.8 Discussion . . . . . . . . . . . . . . . . . 185

9 Multi-View Identification $\quad 187$

9.1 View-based Models . . . . . . . . . . . . . . . . . . . . . 189

9.2 The Role of Prior Knowledge . . . . . . . . . . . . . . 191

9.3 View Correspondence in Identification . . . . . . . . . . . . 191

9.3.1 Learning Linear Shape Models . . . . . . . . . . . . . 192

9.3.2 Nonlinear Shape Models . . . . . . . . . . . . . . . 195

9.4 Gencralisation from a Single View . . . . . . . . . . . 199

9.4.1 Identity by Linear Combination . . . . . . . . . . . 199

9.4.2 Identity by Similarity to Prototype Views . . . . . . 201

9.5 Generalisation from Multiple Views . . . . . . . . . . 203

9.6 Biological Perspectives . . . . . . . . . . . . . . 205

9.7 Discussion . . . . . . . . . . . . . . . . . 207

10 Identifying Moving Faces 209

10.1 Biological Perspectives . . . . . . . . . . . . . . . . . 210

10.1.1 Learning and Identity Constancy . . . . . . . . . . 210

10.1.2 The Effect of Temporal Order of Pose on Learning . . . 210

10.1.3 The Effect of Motion on Familiar Face Identification . . 211

10.2 Computational Theories of Temporal Identification . . . . . . 213 
10.2.1 Atemporal Representation . . . . . . . . . . . . . 213

10.2.2 Spatio-temporal Signatures . . . . . . . . . . . . 214

10.3 Identification using Holistic Temporal Trajectories . . . . . 215

10.4 Identification by Continuous View Transformation . . . . . . 218

10.5 An Experimental System . . . . . . . . . . . . . . . 219

10.6 Discussion . . . . . . . . . . . . . . . 225

Part IV Perception in Context 227

11 Perceptual Integration 229

11.1 Sensory and Model-based Vision . . . . . . . . . . . 230

11.2 Perceptual Fusion . . . . . . . . . . . . . . 231

11.3 Perceptual Inference . . . . . . . . . . . . . . 236

11.3.1 Inference using Hidden Markov Models . . . . . . . . 240

11.3.2 Closed-loop Perceptual Control . . . . . . . . . . . . 241

11.4 Vision as Cooperating Processes . . . . . . . . . . . . . 242

11.4.1 Visual Attention and Grouping . . . . . . . . . . 242

11.4.2 Face Detection, Tracking and Identification . . . . . . 245

11.5 Biological Perspectives . . . . . . . . . . . . . . 247

11.6 Discussion . . . . . . . . . . . . . . . . . 250

12 Beyond Faces 253

12.1 Multi-modal Identification . . . . . . . . . . . . . 254

12.2 Visually Mediated Interaction . . . . . . . . . . . 255

12.3 Visual Surveillance and Monitoring . . . . . . . . . . . . 258

12.4 Immersive Virtual Reality . . . . . . . . . . . . . . . . 260

12.5 Visual Database Screening . . . . . . . . . . . . . . . 261

$\begin{array}{ll}\text { PART V APPENDICES } & 265\end{array}$

A Databases $\quad \mathbf{2 6 7}$

A.1 Database Acquisition and Design . . . . . . . . . 267

A.1.1 Intrinsic Experimental Variables . . . . . . . . . 268

A.1.2 Extrinsic Experimental Variables . . . . . . . . . . . 268

A.2 Acquisition of a Pose-labelled Database . . . . . . . . . 269

A.2.1 Using Artificial Markers . . . . . . . . . . . . . . 269

A.2.2 Using Sensors and a Calibrated Camera . . . . . . . 271 
A.3 Benchmarking . . . . . . . . . . . . . . . . 273

A.4 Commercial Databases . . . . . . . . . . . . . 275

A.5 Public Domain Face Databases . . . . . . . . . . . 277

A.6 Discussion . . . . . . . . . . . . . . . . . . 279

B Commercial Systems $\quad \mathbf{2 8 1}$

B.1 System Characterisation . . . . . . . . . . . . . . . 282

B.2 A View on the Industry . . . . . . . . . . . 283

B.2.1 Visionics Corporation . . . . . . . . . . . . 284

B.2.2 Miros Inc. . . . . . . . . . . . . . . . . . 286

B.2.3 VisionSpheres Technologies . . . . . . . . . . . 287

B.2.4 Eigenface-based Systems: Viisage Technologies, Intelligent Verification Systems and Facia Reco . . . . . . 289

B.2.5 Systems based on Facial Feature Matching: Plettac Electronic Security GmbH, ZN Bochum GmbH and Eyematic Interfaces Inc. . . . . . . . . . . . . . . 291

B.3 Discussion . . . . . . . . . . . . . . . . . . . . . . . 295

C Mathematical Details $\quad 297$

C.1 Principal Components Analysis . . . . . . . . . . . . . . . 297

C.2 Linear Discriminant Analysis . . . . . . . . . . . . . . 298

C.3 Gaussian Mixture Estimation . . . . . . . . . . . . . 300

C.3.1 Expectation-maximisation . . . . . . . . . 300

C.3.2 Automatic Model Order Selection . . . . . . . . . 301

C.3.3 Adaptive EM for Non-stationary Distributions . . . . 302

C.4 Kalman Filters . . . . . . . . . . . . . . . . . . . . 304

C.4.1 Zero-order Prediction . . . . . . . . . . . . 304

C.4.2 First-order Prediction . . . . . . . . . . . 305

C.5 Bayesian Belief Networks . . . . . . . . . . . 306

C.6 Hidden Markov Models . . . . . . . . . . . . . . . . . . . 309

C.7 Gabor Wavelets . . . . . . . . . . . . . . . . . . . 312

$\begin{array}{ll}\text { Bibliography } & 315\end{array}$

$\begin{array}{ll}\text { Index } & 339\end{array}$ 


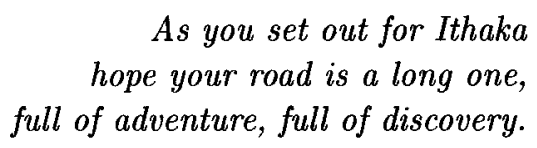

- C.P. Cavafy, Ithaka 
This page is intentionally left blank 


\section{Preface}

Face recognition is a task that the human vision system seems to perform almost effortlessly, yet the goal of building computer-based systems with comparable capabilities has proven to be difficult. The task implicitly requires the ability to locate and track faces in scenes that are often complex and dynamic. Recognition is difficult because of variations in factors such as lighting conditions, viewpoint, body movement and facial expression. Although evidence from psychophysical and neurobiological experiments provides intriguing insights into how we might code and recognise faces, its bearings on computational and engineering solutions are far from clear. In this book, we describe models and algorithms that are capable of performing face recognition in a dynamic setting. The key question is how to design computer vision and machine learning algorithms that can operate robustly and quickly under poorly controlled and changing conditions.

The study of face recognition has had an almost unique impact on computer vision and machine learning research at large. It raises many challenging issues and provides a good vehicle for examining some difficult problems in vision and learning. Many of the issues raised are relevant to object recognition in general. In particular, face recognition is not mercly a problem of pattern recognition of static pictures; it implicitly but crucially invokes many more general computational tasks concerning the perception of moving objects in dynamic and noisy scenes. Consideration of face recognition as a problem in dynamic vision is perhaps both novel and important. The algorithms described in this book have numerous potential applications in areas such as visual surveillance, multimedia and visually mediated interaction.

There have been several books and edited collections about face recognition written over the years, primarily for studies in cognitive psychology or related topics $[38,39,41,42,43,46,378]$. In more recent years, there has been an explosion of computer vision conferences and special work- 
shops dedicated to the recognition of human faces and gestures $[162,163$, $164,165,166,167,168,365]$. Surprisingly, however, there has been no book that provides a coherent and unified treatment of the issue from a computational and systems perspective. We hope that this book succeeds in providing such a treatment of the subject useful for both academic and industrial research communities.

This book has been written with an emphasis on computationally viable approaches that can be readily adopted for the design and development of real-time, integrated machine vision systems for dynamic object recognition. We present what is fundamentally an algorithmic approach, although this is founded upon recent theories of visual perception and learning and has also drawn from psychophysical and neurobiological data.

We address the range of visual tasks needed to perform recognition in dynamic scenes. In particular, visual attention is focused using motion and colour cues. Face recognition is attempted by a set of co-operating processes that perform face detection, tracking and identification using view-based, 2D face models with spatio-temporal context. The models are obtained by learning and are computationally efficient for recognition. We address recognition in realistic and therefore poorly constrained conditions. Computations are essentially based on a statistical decision making framework realised by the implementation of various statistical learning models and neural networks. 'The systems described are robust to factors such as changing illumination, poor resolution and large head rotations in depth. We also describe how the visual processes can co-operate in an integrated learning system.

Overall, the book explores the use of visual motion detection and estimation, adaptable colour models, active and animate vision principles, statistical learning in high-dimensional feature spaces, vector space dimensionality reduction, temporal prediction models (e.g. Kalman filters, hidden Markov models and the Condensation algorithm), spatio-temporal context, image filtering, linear modelling techniques (e.g. principal components analysis (PCA) and linear discriminants), non-linear models (e.g. mixture models, support vector machines, nonlinear PCA, hybrid neural networks), spatio-temporal models (e.g. recurrent neural networks), perceptual integration, Bayesian inference, on-line learning, view-based representation and databases for learning. 
We anticipate that this book will be of special interest to researchers and academics interested in computer vision, visual recognition and machine learning. It should also be of interest to industrial research scientists and managers keen to exploit this emerging technology and develop automated facc and human recognition systems for a host of commercial applications including visual surveillance, verification, access control and video-conferencing. Finally, this book should be of use to post-graduate students of computer science, electronic and systems engineering and perhaps also of cognitive psychology.

The topics in this book cover a wide range of multi-disciplinary issues and draw on several fields of study without requiring too deep an understanding of any one area in particular. Nevertheless, some basic knowledge of applied mathematics would be useful for the reader. In particular, it would be convenient if one were familiar with vectors and matrices, eigenvectors and eigenvalues, some linear algebra, multivariate analysis, probability, statistics and elementary calculus at the level of 1st or 2nd year undergraduate mathematics. However, the non-mathematically inclined reader should be able to skip over many of the equations and still understand much of the content.

\author{
Shaogang Gong \\ Stephen McKenna \\ Alexandra Psarrou
}

October 1999, London and Dundee 


\section{Nomenclature}

Vectors are column vectors, i.e. $\left.\mathbf{x}^{\mathrm{T}} \equiv \mid x_{1} x_{2} \ldots x_{N}\right]$. A list is written as $\left(x_{1}, x_{2}, \ldots x_{N}\right)$ while a set or sequence is denoted by $\left\{x_{1}, x_{2}, \ldots x_{N}\right\}$. Furthermore, $\left\{\left(x_{1}, y_{1}\right),\left(x_{2}, y_{2}\right), \ldots\left(x_{N}, y_{N}\right)\right\}$ denotes a set or sequence of lists. Other commonly used symbols in the book are:

$N, n \quad$ Input space dimensionality, index

$M, m \quad$ Number of examples, index

$C, c \quad$ Number of classes, index

$K, k \quad$ Number of basis functions or discrete states, index

$T, t \quad$ Number of time-steps (frames), time variable or index

$i, j \quad$ Indices

$x, y \quad$ Coordinates in the image plane

$s, r, a \quad$ Scale, orientation and aspect ratio in the image plane

$\theta, \phi \quad$ Tilt and yaw (rotation out of the image plane)

$\kappa, \xi \quad$ A constant, an error variable

$\lambda$

Eigenvalue, wavelength or a hidden Markov model

$\sigma, \boldsymbol{\mu}, \boldsymbol{\Sigma}$

$\mathbf{u}, \boldsymbol{\alpha}$

Standard deviation, mean vector and covariance matrix

a

Eigenvector, a representation vector

$\mathbf{x}, \mathbf{q}, \mathbf{y}$

Parameter vector

$\tilde{\mathbf{x}}, \mathbf{x}^{*}$

Observation, state and interpretation label vectors

$\gamma, \boldsymbol{\xi}$

An approximation to $\mathbf{x}$, a prediction of $\mathbf{x}$

$\mathcal{X}, \mathcal{Q}, \mathcal{Y}$

Rotation in depth (both yaw and tilt)

Sets or sequences of observations, states, interpretations

$\mathcal{O}, \mathcal{S}$

$|\boldsymbol{A}|, \boldsymbol{A}^{\mathrm{T}}, \boldsymbol{I}$ $\Re^{N}$

Object, scene background

Determinant and transpose of matrix $\boldsymbol{A}$, identity matrix $\mathrm{N}$-dimensional space of reals

$f(\cdot), d(\cdot), h(\cdot)$

A function, a distance function and a similarity function $P(\cdot), E(\cdot), L(\cdot)$

$p(\cdot)$

Probability, expectation and likelihood

$G(\cdot), \phi(\cdot)$

$I(\cdot)$

$\mathcal{E}(\cdot), \mathcal{L}(\cdot), \mathcal{R}(\cdot)$

$\|\mathbf{x}\|$

$\ln$

$\otimes$
Probability density function (PDF)

Gaussian function (normal distribution), kerncl function Intensity function (monochrome image)

Error function, loss function and risk functional

$L_{2}$ norm of $\mathbf{x}$ (Euclidean length)

Logarithm to base $e$

Convolution 


\section{Acknowledgements}

We should like to express our considerable gratitude to the many fine people who have helped us in the process of writing this book. The experiments described herein would not have been possible without the work of PhD students, research assistants and post-docs at QMW and the University of Westminster. In particular, we want to thank J.J. Collins, Yongmin Li, Peter Loft, Jeffrey Ng, Eng-Jon Ong, Yogesh Raja, Sami Romdhani, Jamie Sherrah, Jonathan Tanner, Fernando de la Torre, Paul Verity and Michael Walter for their contributions to this work. We are indebted to Hilary Buxton, Robert Koger and Dennis Parkinson, all of whom read a draft carefully and gave us many insightful comments and suggestions.

We should also like to thank the anonymous reviewers for their constructive feedback as well as John Navas at Imperial College Press and R. Sankaran at World Scientific Publishing for the kind help we have received in the final stages of preparing this book. The book was typeset using IATEX.

Our thanks are also due to Wolfgang Konen and Elke Sassmannshausen at ZN GmbH in Bochum, Johannes Steffens at Eyematic Interfaces Inc., James Kottas at Miros Inc. and Martin Levine at VisionSphere Technologies Inc. for kindly responding to our enquiries concerning their companies' products which are summarised in Appendix B.

We gratefully acknowledge the financial support that we have received over the years from UK EPSRC, EU ESPRIT and HCM networks, the BBC, BT and Safehouse Technologies Inc. Finally, we should like to thank our farnilies and friends for all their support. 
Perchance the best chance of reproducing the ancient Greek temperament would be to cross the Scots with the Chinese.

Murray Christopher Grieve (Hugh McDiarmid), Lucky Poet 